Herzschr Elektrophys 2013 $\cdot 24: 72-74$

DOI 10.1007/s00399-013-0257-7

Online publiziert: 11. April 2013

๑) Springer-Verlag Berlin Heidelberg 2013

\author{
G. Fröhlig ${ }^{1} \cdot$ W. Fischer ${ }^{2} \cdot$ U. Wiegand ${ }^{3}$ \\ ${ }^{1}$ Homburg, Deutschland \\ ${ }^{2}$ Innere Abteilung Krankenhaus Peißenberg, Peißenberg, Deutschland \\ ${ }^{3}$ Klinik für Kardiologie, Pneumologie und Internistische Intensivmedizin, \\ Sana-Klinikum Remscheid, Remscheid, Deutschland
}

\title{
Schrittmacher und ICDs
}

\section{Prognose subklinischer Vorhofarrhythmien (ASSERT)}

Die Speicher moderner Herzschrittmacher und ICDs liefern zahlreiche Informationen, so auch die Detektion klinisch inapparenten Vorhofflimmerns oder -flatterns. Es war bislang unklar, ob der Nachweis solcher Arrhythmien einen prognostischen Wert hat. Dies wurde durch die ASSERT-Studie [1] eindrucksvoll belegt.

In die Studie wurden 2580 Patienten mit neu implantiertem Schrittmacher $(n=2451)$ oder ICD ( $n=129)$ eingeschlossen, die älter als 65 Jahre waren, keine Vorgeschichte von Vorhofflimmern/-flattern $>5$ min aufwiesen und nicht unter oraler Antikoagulation standen [2]. Drei Monate nach Einschluss wurden die Schrittmacherspeicher nach symptomfreien atrialen Tachyarrhythmien ( $\mathrm{f}>190 / \mathrm{min},>6 \mathrm{~min}$ Dauer) durchsucht. Zusätzlich wurde bei Herzschrittmacherpatienten ein kontinuierlicher atrialer Überstimulationsalgorithmus randomisiert aktiviert oder inaktiv belassen.

Bei 261 Patienten (10,1\%) fanden sich drei Monate nach Studieneinschluss subklinische atriale Arrhythmien. Bei diesen Patienten trat im weiteren Verlauf ein symptomatisches Vorhofflimmern/-flattern mehr als 5-mal häufiger auf als in der Kontrollgruppe (HR 5,56 [3,78-8,17]). Auch das Risiko eines ischämischen Schlaganfalls oder einer systemischen Embolie war bei diesen Patienten mit $1,7 \%$ pro Jahr um mehr als das Doppelte erhöht (HR 2,49 [1,28-4,85]). Der Vorhersagewert der subklinischen atrialen Arrhythmien war vom CHADS2-Score abhängig. Bei einem CHADS2-Score $>2$ betrug das Schlaganfallrisiko 3,8\% pro Jahr. Die permanente atriale Overdrive-Stimu- lation beeinflusste die Vorhofflimmerinzidenz nicht. Die Studie schließt damit das Kapitel der atrialen Schrittmacherstimulation zur Vorhofflimmerprävention endgültig ab.

Die ASSERT-Studie belegt eindrucksvoll die Bedeutung kurzer atrialer Arrhythmieepisoden in den Schrittmacherspeichern. Methodenbedingt - die Studie war als Vorhofflimmerpräventionsstudie geplant - wurden diese nur in den ersten drei Monaten nach Studieneinschluss und nicht kontinuierlich erfasst, sodass der Vorhersagewert der Arrhythmieepisoden wahrscheinlich unterschätzt wurde. Allerdings handelt es sich nicht um eine Interventionsstudie. Wir wissen daher nicht, ob eine orale Antikoagulation der Patienten mit symptomfreien Episoden einen Nettonutzen erzielt hätte.

Da uns solche Daten in den nächsten Jahren aber nicht zur Verfügung stehen werden, bleibt es derzeit bei diesen Patienten bei einer individuellen Entscheidung zur Antikoagulation, die aber durch die Studie zumindest für Patienten mit erhöhtem Embolierisiko unterstützt wird.

\section{CRT bei AV-Block (Block-HF)}

Seit DAVID und MOST ist die Frage virulent, ob Patienten mit hohem Stimulationsbedarf in der Kammer weniger Schäden an ihrer Ventrikelfunktion erleiden, wenn sie statt klassisch AV-sequenziell mit einem biventrikulären System versorgt werden. Obwohl seit Jahren erwartet, ist die Block-HF-Studie 2012 noch nicht publiziert worden, hat aber immerhin auf der AHA-Tagung im November als erste Untersuchung zum Thema vorläufige Daten geliefert [3].
Die Studienpopulation umfasst 691 von ursprünglich 918 erfassten Patienten. 95 Patienten wurden wegen mangelnder Einschlusskriterien (stimulationsbedürftiger AV-Block, LVEF $\leq 50 \%$, Herzinsuffizienz (HF) NYHA I-III, keine KlasseI-Indikation zu CRT, erstes Rhythmusimplantat) ausgeschlossen, 14 wegen Widerrufs des Einverständnisses, 51 mit fehlgeschlagener Implantation und 67 ohne Randomisierung. Nach Implantation eines CRT-Systems (CRT-P: 484; CRT-D: 207) werden 349 Patienten zu bi- (BiV), 342 zu rechtsventrikulärer (RV) Stimulation randomisiert. Mit CRT-D und CRT-P versorgte Patienten unterscheiden sich nach mittlerer LVEF ( $33 \pm 8 \%$ vs. $44 \pm 7 \%$ ) und Prävalenz einer koronaren Genese (61 vs. $39 \%$ ). CRT-D geht mit weniger ausgeprägter AV-Blockierung und intensiverer Herzinsuffizienzmedikation einher. Es werden keine Angaben zur Signifikanz der demografischen Differenzen gemacht. Das mittlere Follow-up beträgt $36(\mathrm{BiV})$ bzw. 38 Monate (RV) mit einer Compliance von $94 \%$ aller Visiten. Rund $35 \%$ der Klientel erreicht weniger als 2 Jahre Beobachtungszeit. Die Ergebnisse sind in - Tab. 1 zusammengefasst.

Bei getrennter Analyse verliert die CRT-D-Gruppe die Signifikanz allfälliger Unterschiede.

Obwohl alle Patienten ein CRT-System haben, imponieren die Komplikationen beim biventrikulären Betrieb von Defibrillatoren besonders und erreichen 38 vs. $24 \%$ bzw. 30 vs. $12 \%$, wenn Gesamtsystem- bzw. Aggregatprobleme zwischen CRT-D und ICD-RV verglichen werden.

In Würdigung der vorläufigen Daten lässt sich den Autoren folgend als Fazit ziehen: 
Tab. 1 Klinische Endpunkte bei BiV versus RV [3]

\begin{tabular}{|ll|}
\hline Endpunkt & HR $(95 \%$ Cl) \\
\hline Primärer Endpunkt (Mortalität, HF-Notfallbehandlung, $\uparrow$ LVESVI $\geq 15 \%$ & $0,74(0,60-0,90)$ \\
\hline Mortalität jedweder Ursache, notfallmäßige HF-Behandlung & $0,73(0,57-0,92)$ \\
\hline Mortalität jedweder Ursache, Hospitalisation wegen Herzinsuffizienz & $0,78(0,61-0,99)$ \\
\hline Hospitalisation wegen Herzinsuffizienz & $0,70(0,52-0,93)$ \\
\hline Mortalität & $0,83(0,61-1,14)$
\end{tabular}

Stärken der Studie:

- Erste große, prospektiv randomisierte, doppelt verblindete Langzeitstudie, die

- einen Unterschied in klinischen Endpunkten zwischen BiV- und RV-Stimulation bei Patienten mit AV-Block und unterschiedlichen Graden der Herzinsuffizienz zeigt.Allerdings beruht der Unterschied im primären Endpunkt vornehmlich auf dem weichen Endpunkt der Notfallbehandlung wegen Herzinsuffizienz (ohne die Bedingung der stationären Aufnahme), während nach der eher kurzen Beobachtungszeit kein Überlebensvorteil besteht.

Ihre Schwächen:

- Lange und zögerliche Einschlussphase (Studiendesign 2007 bereits publiziert),

- reduzierte Aussagekraft durch Zensierung wegen fehlender LVESVI-Daten $(154 / 691=22 \%)$ und

- Ungleichgewicht in den CrossoverRaten $(\mathrm{BiV} \rightarrow \mathrm{RV}: 4,6 \%$; RV $\rightarrow \mathrm{BiV}$ : $24,6 \%)$.

\section{Magnetresonanztomo- graphie- (MR-)Sicherheit bei aktiven Implantaten}

Grundsätzlich gibt es bei dem Thema Magnetresonanztomographie- (MR-)Sicherheit bei aktiven Implantaten (Herzschrittmacher, Defibrillator oder $\mathrm{CRT}^{1}$-Gerät einschließlich Elektroden) bei der Übersicht der Literatur im Jahre 2012 keine wesentlichen neuen Erkenntnisse. Das für die Sicherheit von implantierbaren Herzschrittmachern, Defibrillatoren und CRTGeräten in Deutschland zuständige Gremium „Aktiv betriebene Implantate“ der DKE (Deutsche Kommission für Elektro-

${ }^{1}$ Kardiale Resynchronisationstherapie. technik Elektronik Informationstechnik im DIN und VDE) befasste sich speziell mit der aktuellen Problematik und gab ein Positionspapier heraus, das als Kurzfassung vorliegt [4] und die gegenwärtige Situation zusammenfasst:

„MR-sichere aktive Implantate " gibt es nicht. Man unterscheidet deshalb „bedingt MR-sichere Systeme“ und "nicht MR-sichere, konventionelle Systeme“.

\section{Nicht MR-sichere konventionelle Systeme}

Bei den „nicht MR-sicheren konventionellen Systemen“ sind sich die Hersteller einig, dass eine MR-Untersuchung kontraindiziert ist. Je nach Bauart des MRGeräts und der Funktion des Implantats muss mit unterschiedlichen Komplikationen gerechnet werden, wie Umschalten in den Magnetmodus mit der Möglichkeit von Kammerflimmern, komplette Abschaltung der Tachykardieerkennung, Beschädigung des Reedschalters (die Gefahren des Magnetmodus bleiben permanent oder zumindest für Stunden erhalten, das Implantat kann nicht mehr programmiert werden), Störmodus mit festfrequenter Stimulation, Inhibition der Stimulation, inadäquate Schockabgaben, „run-away“ mit hochfrequenter Stimulation, komplettem Reset, Zerstörung der Elektronik mit Therapieverlust etc., aber auch Erwärmung der Sondenspitze mit Koagulation des umgebenden Gewebes bzw. Stimulationsverlust. Ob eine der Komplikationen auftritt, lässt sich nicht vorhersagen. Manche Risiken können in Zusammenarbeit von Kardiologen und Radiologen reduziert werden, Todesfälle und lebensbedrohliche Komplikationen sind seit dem Jahr 2000 nicht mehr beschrieben worden.

Sollte bei Patienten mit nicht sicheren konventionellen Schrittmacher-/ICD-/ CRT-Systemen eine MR-Untersuchung vital indiziert oder unvermeidbar sein, muss der Patient über die fehlende Zulassung und die möglichen Nebenwirkungen dieser Untersuchung gründlich aufgeklärt und das Nutzen-Risiko-Verhältnis gemeinsam sorgfältig abgewogen werden. Das Aufklärungsgespräch und die Einverständniserklärung müssen genau dokumentiert werden.

\section{Bedingt MR-sichere Systeme}

Wenn ein Hersteller seinem Produkt MR-Fähigkeit bescheinigt, dann ist diese an ganz strenge Randbedingungen geknüpft. Deswegen werden diese Implantate (Aggregate und Elektroden) auch nur als bedingt MR-sicher bezeichnet und behalten Risiken, über die ein Patient aufgeklärt werden muss. Die Randbedingungen unterscheiden sich je nach Hersteller und Modell (entsprechend dem Zulassungsverfahren) und betreffen speziell den Kardiologen und Radiologen. Unter anderem gilt (Stand 2012):

- Dem Patienten wurde das bedingt MR-sichere Stimulationssystem mit den dazugehörigen MR-tauglichen Elektroden implantiert.

- Das Aggregat befindet sich während der Untersuchung im MR-Schutzmodus.

- Der Patient zeigt zum Zeitpunkt der MR-Untersuchung keine erhöhte Körpertemperatur oder gestörte Wärmeregulation.

- Mindestens sechs Wochen sind seit der Implantation und/oder einer Elektrodenkorrektur oder chirurgischen Änderung des bedingt MR-sicheren Aggregats vergangen.

- Es sind neben dem bedingt MR-sicheren Stimulationssystem keine weiteren Geräte für das Herz, sowie Komponenten oder Zubehörteile (z. B. stillgelegte Elektroden) implantiert.

- Die Restbatteriekapazität des Aggregats beträgt mindestens $30 \%$.

- Die MR-Magnetstärke ist gegenwärtig durch genau 1,5 T definiert.

- Maximaler räumlicher Gradient in $\mathrm{T} / \mathrm{m}$ und max. Anstiegssteilheit unterhalb eines Wertes in T/m/s sind den individuellen Grenzwerten des Implantatherstellers zu entnehmen. 
- Nur horizontale, geschlossene Protonen-(1H-)Systeme sind erlaubt

- Grenzen der spezifischen Absorptionsrate (SAR) und Grenzen des Gradientenfelds müssen für Scansequenzen im normalen Betriebsmodus überwacht und eine maximale MRUntersuchungsdauer darf nicht überschritten werden.

- Während der MR-Untersuchung ist die Sauerstoffsättigung des Patienten mittels Pulsoximeter und/oder die Herztätigkeit mit einem EKG zu überwachen.

- Während der Untersuchung müssen ein externer Defibrillator und medizinisches Personal, das in Herz-Lungen-Wiederbelebung ausgebildet ist, zwingend vor Ort sein.

- Scanausschlusszonen (1 Hersteller) sind zu beachten.

- Nach der MR-Untersuchung ist die Reprogrammierung des Aggregats aus der MR-Betriebsart in seine „Normalfunktion“ sowie die Kontrolle der Elektroden und Geräteparameter mittels Programmiergerät notwendig.

Neben der Darstellung der aktuellen Situation weist das Positionspapier im Hinblick auf die gegenwärtige Literatur besonders auf die genaue Beachtung der aktuellen umfangreichen Zulassungsbeschränkungen der „bedingt MR-sicheren Implantate" hin, z. B, dass diese Geräte zurzeit nur die Zulassung für genau 1,5-T-MR-Geräte haben, nicht mehr, aber auch nicht weniger Tesla (wie es etwas ungenau in Publikationen dargestellt wird), also auch nicht für 0,5-T-Geräte. Zugleich geht das Gremium auch darauf ein, dass mit Erfahrungsberichten allein nicht belegt werden kann, dass eine MR-Untersuchung konventioneller Aggregate bei Einhaltung eines bestimmten prozeduralen Managements sicher durchgeführt werden kann. Wenn eine bestimmte Zahl von Untersuchungen komplikationslos verlaufen sei, könne man daraus nicht auf eine genügende Sicherheit schließen, da die Mechanismen, warum es zu Zwischenfällen gekommen ist und zu Zwischenfäl- len kommen kann, nicht umfassend bekannt sind.

Als Fazit ergibt sich, dass eine generelle MR-Sicherheit von aktiven Implantaten gegenwärtig nicht gegeben ist. Wenn sich ein Arzt dafür entscheiden will, eine solche Untersuchung durchzuführen, kann er das nur unter Risikoabwägung bei Einhaltung aller Sicherheitsmaßnahmen und bei einer ins Detail gehenden Aufklärung des Patienten tun. Im Unterschied zu den „nicht MR-sicheren konventionellen Systemen" befindet sich der Arzt (z. B. Kardiologe/ Radiologe) bei den seit 2008 auf dem Markt befindlichen „bedingt MR-sicheren Implantaten“ nicht mehr im OffLabel-Bereich und ist damit juristisch deutlich besser abgesichert, unter der Voraussetzung, dass er sich genau an die aktuellen für das jeweilige Schrittmacher-, ICD-, CRT-System geltenden umfangreichen Zulassungsbeschränkungen, bzw. Rahmenbedingungen hält. Aber auch in diesem Falle sind eine entsprechende Aufklärung und das sorgfältige Abwägen des Nutzen-Risiko-Verhältnisses unverzichtbar.

\section{Korrespondenzadresse}

\section{Prof. Dr. G. Fröhlig}

Kraftwerkstrasse 24, 66424 Homburg,

Deutschland

ingfro@kabelmail.de

\section{Literatur}

1. Healy FS, Connolly SJ, Gold MR et al (2012) Subclinical atrial fibrillation and the risk of stroke. $\mathrm{N}$ Engl J Med 360:120-129

2. Hohnloser SH, Capucci A, Fain E et al (2006) A symptomatic atrial fibrillation and stroke evaluation in pacemaker patients and the atrial fibrillation reduction atrial pacing trial (ASSERT). Am Heart J 152:442-447

3. Curtis A et al (2012) Biventricular versus right ventricular pacing in patients with left ventricular dysfunction and atrioventricular block. Preliminary results. Late-Breaking Clinical Trials, LBCT.06: Management of LV Dysfunction: Devices and Drugs. AHA

4. Fischer W, Lampadius MS, Mols R, Schaefers $G$ (2013) MRT bei aktiven Implantaten - Generelle Sicherheit gibt es nicht. Dtsch Ärztebl 110:A555A556 